\title{
EDITORIAL
}

\section{Funding OF RESEARCH}

AT A RECENT MEETING which I attended abroad, a senior officer of a funding agency spoke on the policy of his organization in support of research. At one point in the presentation he made the statement that he was quite prepared to support research in anaesthesia but not research by anaesthetists. My first reaction was that this seemed to confirm a lingering suspicion of discrimination in the allocation of research funds, a view to which many anaesthetists subscribe. But upon more mature reflection it occurred to me that perhaps I had failed to take into account the fact that he had spoken before an audience of anaesthetists and had his audience been different he might perhaps have said that he was prepared to support research in medicine but not necessarily research by physicians. In any case the statement as it was made might merit some analysis because I am reasonably certain that funding agencies in Canada are motivated by quite similar considerations.

I believe what the man tried to convey was that the limited funds at his disposal were available only to serious well-trained researchers in anaesthesia and could no longer be made available to every amateur who feels the urge to do "some research," likely with an eye on having to show evidence of such activities to qualify for a senior appointment, a promotion, or some such professional advancement.

One need only review the world literature to realize that much of what is published is devoid of any real lasting merit, does not exhibit much originality of thought, and often does little to shed light on new problems. The literature of anaesthesia is no exception. Much is repetitious and only confirms what has been found by others before. Some other work is poorly controlled or badly conceived to start with. The really good work most often comes from men who have had special research training, even if much of their daily professional activities remain in the clinical field.

As in so many other human activities, research too has become complicated and the sophisticated methodology involved requires special training and experience. This places new demands on the young people who intend to embark on academic careers since the days are gone when, after completion of clinical training alone, they could embark on a career which might also include research activities. It is fortunate that individuals are now coming along in increasing numbers who have acquired truly professional expertise in research, in addition to first-class clinical training. Unfortunately their numbers are still far too small, and many more are needed before our friend, who started it all, may feel confident to entrust "anaesthetists" with his carefully husbanded and limited resources.

May 13, 1974

Gordon M. WyanT, M.D.

\section{Fondations ET RECHERCHE MÉdicale}

LORS D'Un CONGRès RÉCENT, à l'extérieur du pays, j’ai entendu l'un des administrateurs séniors d'une fondation, exprimer les politiques de sa fondation, vis à vis la 
Recherche. Au cours de son exposé il déclara être prêt à supporter la Recherche en Anesthésie, mais non la Recherche conduite par des anesthésistes. Ma première réaction fut que ceci confirmait mes "soupçons" de discrimination contre notre spécialité dans la distribution des fonds de Recherche. C'est une opinion partagée par de nombreux anesthésistes.

A la réflexion, je réalisai cependant qu'il avait prononcé cet exposé devant un groupe d'anesthésistes et qui si son auditoire avait été différent, il aurait peut être dit que sa fondation était disposée à contribuer à la recherche en médecine, mais pas nécessairement à une recherche faite par des médecins. De toutes façons, l'énoncé de cet administrateur, prête à réflexion car je suis à peu près certain que les fondations canadiennes ont des opinions du même genre.

Je crois que l'administrateur voulait nous faire réaliser que les ressources limitées à sa disposition n'étaient disponibles que pour des chercheurs sérieux et bien formés, que ces ressources ne pourraient plus être versées au premier amateur qui sentirait le besoin de faire une recherche quelconque, possiblement dans le désir de démontrer qu'il fait de la Recherche, ce qui l'aidera à se qualifier en vue d'une promotion, d'une nomination.

Nous n'avons qu’à regarder ce qui est publié pour constater la grande quantité de publications sans mérite réel, sans originalité de pensée, et ne contribuant que très peu à apporter de la lumière sur de nouveaux problèmes. La littérature d'anesthésie ne fait pas exception à cet égard. De nombreuses publications ne sont que des répétitions et ne confirment que ce qui a déjà été énoncé par d'autres.

D'autres travaux sont mal contrôlées ou pauvrement conçus dès le départ. Les travaux réellement valables viennent le plus souvent de gens qui ont déjà une formation spéciale en recherche, même si une grande partie de leurs activités professionnelles quotidiennes demeure dans le champ clinique.

Comme beaucoup d'autres activités humaines, la recherche est devenue compliquée et la méthodologie sophistiquée qu'elle requiert exige une expérience et un entraînement spéciaux.

Ceci implique de nouvelles exigences pour les jeunes qui désirent s'orienter vers des carrières académiques, puisque le temps est révolu où l'on pouvait entreprendre une carrière qui incluait des activités de recherches, après un entrainement purement clinique. Heureusement, le nombre de ceux qui ont acquis une formation vraiment solide en recherche, en plus de leur entrainement clinique va en s'accroissant. Malheureusement le nombre de ces individus est encore trop petit et leur nombre devra s'accroître de beaucoup avant que notre ami l'administrateur du fonds de recherche puisse confier à des anesthésistes les ressources limitées de sa fondation.

le 13 mai 1974

Gordon M. Wyant, M.D. 